\title{
Compensation of Unknown Time Delay Measurement for Network Visual Servoing System Using Observation Design
}

\author{
Abdeldafia MOHAMMED, Haoping WANG*, Yang TIAN \\ Nanjing University of Science \& Technology, School of Automation, \\ Sino-French International Joint Laboratory of Automatic Control and Signal Processing (LaFCAS), \\ Nanjing, 210094, China. \\ hp.wang@njust.edu.cn (*Corresponding author)
}

\begin{abstract}
In this paper, the sampled and delayed measurements subject to measurement noise are studied under the condition of the time delay being bounded, unknown, and time varying. The proposed observer is called "continuous state observer" and, based on the piecewise continuous hybrid system and on the extended functional observer, is derived to reconstruct the continuous system state without time delay from sampled and delayed measurements subject to measurement noise. The proposed observer has advantages which are represented by simple structure design and robust performance of the measurement noise. Another advantage of the proposed observer is that it considers the time delayed measurement to be a particular disturbance induced by the vision sensor. Moreover, the effectiveness of the continuous state observer is compared with the Chain observer over appropriate example, and the comparison results are established.
\end{abstract}

Keywords: Sampled and delayed measurements, Visual information, State observer, Output disturbance, Hybrid system

\section{Introduction}

In recent years, the vision sensors have further improved the accuracy, the versatility and the drawback of contemporary commercial robots by engaging the visual data in the feedback channel to control objects movement. Thus, utilizing the visual information in the feedback channel directly to the controller is called visual servoing system (VSS). Moreover, employing the visual information in feedback over the network system is called network visual servoing control system (NVSCS) [17, 26].

Even though, using a vision sensor to obtain the visual data confronts with the sampling effects due to the lower sampling rate of the vision sensor. Thus, utilizing visual data with the effect of lowering sampling rate to the controller might affect the performance and may lead to the closed loop instability $[6,16]$. Moreover, using visual data as a feedback signal induces time delay which corresponding to time for image acquisition, image processing, and visual data transmission $[9,21]$. It is worth mentioning that the time delay is the main cause of system instability $[5,13,10]$.

To overcome the problem mentioned above, the standard vision sensor is substituted by a highspeed vision sensor which improves the NVSCS performance and minimizes the sampling effects of the measurement as well as the time delay. However, high-speed vision sensors are very expensive and introduce a great quantity of visual data, which requires specific computational architectures to realize the data processing. In this way, the total cost of the system is prohibitive which is not appropriate for the common of manufacturing applications [12].

Another solution would consist in designing an observer to reconstruct the unavailable continuous system state from the sampled and delayed measurements. During decades, significant research efforts have contributed in the area of systems with sampled and delayed measurements. In [2], the continuous time observer is offered which is designed based on the continuous system model. In [11], the Kalman filter-based observer is introduced for the continuous model of flexible link robot with the sampled and delayed output measurements. It can be noticed that a significant drawback relies on the fact that continuous-time observers neglect the sampling period of the output measurement in the observer design which implies that the observer stability is valid only in the case of the non-large rate of the sampling period. Moreover, another technique is provided based on the Lyapunov-Krasovskii theorem which is called the delayed Luenberger observer and which is presented in $[18,7]$. In [1], two particular classes of global exponential observers are provided for the continuous nonlinear system and the maximum allowable sampling period and the time delay are addressed. In this way, the minimum sampling period must be bigger than the maximum delay, while the time delay is greater or 
smaller than the sampling period in real time. It should be pointed out that all the results in $[18,7$, 1] provide robust stability to plant uncertainties especially in the case of parameters variation. However, to achieve the linear matrix inequalities solution, a difficult software code is needed.

Recently, the hybrid system technique is used in the observation design which allows representing both the continuous and discrete system model in the form of the hybrid system [4]. Taking into account the hybrid system technique, the piecewise continuous observer was proposed to estimate the position of the $\mathrm{x}-\mathrm{y}$ robot $[22,23]$, and then developed in [24]. The piecewise continuous observer is more straightforward compared with other methods like those introduced in $[18,7]$, and it provided better performance than the continuous-time Lyapunov-Krasovskii method. In [15], a hybrid state observer is presented based on the similar hybrid system technique. However, it considers the constant time delay equal to the sampling period. It is worth mentioning that the results in $[22-24,15]$ investigated the linear system without undesirable measurement and the sampling period is less than or equal to the time delay. However, the time delay is greater or smaller than sampling period in practical processes. However, based on hybrid system technique, the problem of the sampled and delayed (unknown time-varying) measurement subject to measurement noise is not considered.

This paper considers the sampled and delayed measurement subject to measurement noise for network visual servoing system. The time delayed in the NVSCS feedback are viewed as the particular type of the output disturbance and the time delay can be compensated by the proposed observer. The main contribution of this work can be concluded as follows: 1) Propose continuous state observer strategies for the particular continuous systems with the problem of sampled and unknown variable delayed measurements subject to measurement noise. 2) The designed observer estimates simultaneously the continuous state without delay and the disturbance from the sampled and delayed measurements. 3) Propose a simple strategy technique of state observation design which is composed of the extended functional observer and the piecewise continuous hybrid system. Furthermore, the proposed scheme is compared with the existing work which is the Chain observer, and the results illustrate the effectiveness of the designed observer.

The rest of this paper is structured as follows: the problem statement of the feedback NVSCS is shown in details in section 2. Section 3 introduces the procedures for the design of the proposed observer method. After that, the proposed observer design stability is investigated through linear matrix inequality as presented in section 4 . Then, the performance of the proposed observer is confirmed through an assessment with the Chain observer, and the obtained results are illustrated in section 5. Finally, section 6 offers the conclusions.

\section{Problem Formulation}

The system of NVSCS that has been considered in this work is described by a continuous-time linear system (CTLS) which is represented as:

$\left\{\begin{array}{l}\dot{x}(t)=A x(t)+B u(t) \\ y=C x(t) \\ \bar{y}(t)=y^{*}(t-d)+v_{k}\end{array}\right.$

where $x(t) \in \mathbb{R}^{n}$ is the plant state, $u(t) \in \mathbb{R}^{r}$ is the plant input, $y(t) \in \mathbb{R}^{m}$ is the unavailable output, $\bar{y}(t)$ is the sampled and delayed available measurement which demonstrates the object position information in sampled form denoted by sampling period $t_{e}$ with sampled notation $*$ and delayed form $d . A \in \mathbb{R}^{n \times n}, B \in \mathbb{R}^{n \times r}, C \in \mathbb{R}^{m \times n}$ are constant matrices. $v_{k}$ is the measurement noise which affects the camera sensor and it is assumed that $v_{k}$ is bounded with the upper bound $\bar{v}$ which is defined as: $\left\|v_{k}\right\| \leq \bar{v}$. In this work, the time delay between the digital camera sensor and the controller is considered; it is assumed that there is no time delay between the controller and the actuator. It is also assumed that $(A, C)$ is observable and $(A, B)$ is controllable. Furthermore, it is supposed that the time delay $d$ is bounded with known values as $0 \leq d(t) \leq d_{m}$ where $d_{m}$ is the maximum time delay.

The main objective of this study is to reconstruct the continuous system state without time delay from sampled and delayed measurement even if the vision sensor has the measurement noise by designing an observer. 


\section{Main Result}

In this section, the state observer design which is called Continuous State Observer (CSO) is introduced to obtain the continuous state without time delay from sampled and delayed measurements subject to measurement noise. The $\mathrm{CSO}$ is constructed from the extended functional observer and the piecewise continuous hybrid system as shown in Figure 1.

By discretizing the system of (1a) and (1b) with $\mathrm{ZOH}$, the discrete time system is derived as:

$x_{k+1}=\Phi x_{k}+\Gamma u_{k}$

$y_{k}=C_{d} x_{k}$

where $x_{k}$ is state in the discrete time domain, and $\Phi=e^{A t_{e}}, \Gamma=\int^{t_{e}} e^{A\left(t_{e}-\tau\right)} B d(\tau)$ and $C_{d}=C$ are corresponding constant matrices in the discrete time domain.

It can be noticed that the pair $\left(\Phi, C_{d}\right)$ and $(\Phi, \Gamma)$ is detectable and stabilizable, respectively and the matrices $\Phi, \Gamma$ and $C_{d}$ satisfy

$\operatorname{rank}\left[\begin{array}{cc}C_{d} & 0 \\ I_{n}-\Phi & -\Gamma\end{array}\right]=n+m$

Before starting the observer design, some Lemmas are needed as follows:

Lemma 1 [27]: The pair $\left(\Phi, C_{d}\right)$ is detectable if and only if it satisfies the following rank condition

$\operatorname{rank}\left[\begin{array}{c}C_{d} \\ \lambda I_{n}-\Phi\end{array}\right]=n \quad$ for all $\lambda \in \mathbb{C}$

Denote the sampled and delayed signal $\bar{y}(t)$ at time instant $k$ as $\bar{y}_{k}$. Thus, equation (1c) can be reformulated as:

$$
\begin{aligned}
\bar{y}_{k} & =y_{k-d_{k}}=C x_{k-d_{k}}+v_{k} \\
& =C x_{k}+\mu_{k}
\end{aligned}
$$

where $\mu_{k}=C x_{k-d_{k}}-C x_{k}+v_{k}$ is the new notation which represents all the disturbances affected at the vision sensor. It considers the measurement noise and camera sensor. For the reason of simplicity $\mu_{k}$ can be called special output disturbance.

Based on the similar unknown input observer approach, the extended state can be defined as

$$
\begin{aligned}
& \bar{x}_{k}=\left[\begin{array}{ll}
x_{k} & \mu_{k}
\end{array}\right]^{T} \\
& \bar{A}=\left[\begin{array}{cc}
\Phi & 0_{n \times m} \\
0_{m \times n} & -\varepsilon I_{m}
\end{array}\right], \bar{E}=\left[\begin{array}{cc}
I_{n} & 0_{n \times m} \\
0_{m \times n} & 0_{m}
\end{array}\right], \bar{B}=\left[\begin{array}{c}
\Gamma \\
0_{m \times r}
\end{array}\right] \\
& \bar{C}=\left[\begin{array}{ll}
C_{d} & I_{m}
\end{array}\right], \bar{P}=\left[\begin{array}{l}
0_{n \times m} \\
\varepsilon I_{m}
\end{array}\right]
\end{aligned}
$$

where $\varepsilon$ is the positive constant which is used to deduce the linear matrix inequality (LMI) feasible problem. $\bar{x}_{k}$ is the augmented system state which contains the system state $x_{k}$ in the discrete time domain and the disturbance noise $\mu_{k}$. It can be noticed that the system (6) is designed based on the descriptor system approach [19], which implies that the term $I_{m}$ in the matrix $\bar{C}$ does not mean that the disturbance is measured at the observer side.

According to the equations (2), (5) and (6) the augmented descriptor model is expressed as follows:

$$
\begin{aligned}
& \bar{E} \bar{x}_{k+1}=\bar{A} \bar{x}_{k}+\bar{B} u_{k}+\bar{P} \mu_{k} \\
& \bar{y}_{k}=\bar{C} \bar{x}_{k}
\end{aligned}
$$

Thus, if the extended state $\bar{x}_{k}$ can be estimated, then it is possible to achieve the state vectors $x_{k}$ and $\mu_{k}$.

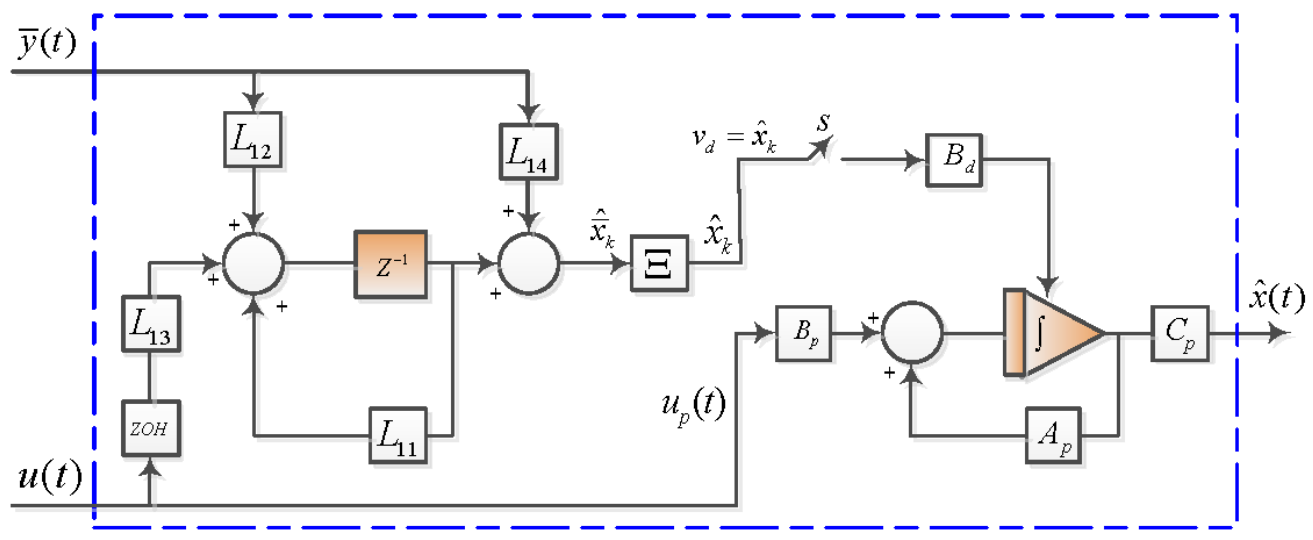

Figure 1. The proposed continuous state observer 
Now it is time to obtain the extended state estimate $\hat{\bar{x}}_{k}$, the extended functional observer which is addressed as:

$\xi_{k+1}=L_{11} \xi_{k}+L_{12} \tilde{y}_{k}+L_{13} u_{k}$

$\hat{\bar{x}}_{k}=\xi_{k}+L_{14} \tilde{y}_{k}$

where $\hat{\bar{x}}_{k} \in \mathbb{R}^{n}$ is the estimation state of $\bar{x}_{k}$, and the auxiliary variable $\xi_{k} \in \mathbb{R}^{n}$ is used. $L_{11}, L_{12}, L_{13}$ and $L_{14}$ are the gains which have to be designed to confirm the estimation error between $\hat{\bar{x}}_{k}$ and $\bar{x}_{k}$ converges to zero asymptotically.

Check the rank of the $\left[\begin{array}{l}\bar{E} \\ \bar{C}\end{array}\right]$ to satisfy the
following condition $\operatorname{rank}\left[\begin{array}{c}\bar{E} \\ \bar{C}\end{array}\right]=\operatorname{rank}\left[\begin{array}{cc}I_{n} & 0_{n \times m} \\ C_{d} & I_{m}\end{array}\right]=n+m$

The auxiliary matrices are used and denoted as $\Psi_{1}$ and $\Psi_{2}$ as follows:

$\left[\begin{array}{ll}\Psi_{1} & \Psi_{2}\end{array}\right]=\left[\begin{array}{l}\bar{E} \\ \bar{C}\end{array}\right]^{+}$

where $\mathrm{O}^{+}$represents pseudoinverse of $\mathrm{O}$ satisfying $\mathrm{O}^{+}=\left(\mathrm{O}^{T} \mathrm{O}\right)^{-1} \mathrm{O}^{T}$.

$\Psi_{1} \bar{E}+\Psi_{2} \bar{C}=I_{n+m}$

Therefore, to verify equation (11) the matrices $\Psi_{1}$ and $\Psi_{2}$ are selected as:

$\Psi_{1}=\left[\begin{array}{cc}I_{n} & 0_{n \times m} \\ -C_{d} & I_{m}\end{array}\right], \quad \Psi_{2}=\left[\begin{array}{c}0_{n \times m} \\ I_{m}\end{array}\right]$

In order to design the observer parameters $L_{11}, L_{12}, L_{13}$ and $L_{14}$ that ensure the estimation error of the state estimation settles down to the origin. In order to design the observer parameters, the following Theorem is used.

Theorem 1: The estimation error for estimating system state in (7) is asymptotically stable if and only if there exists a matrix $N$ such that $\Psi_{1} \bar{A}-N \bar{C}$ is Schur and the observer's gains satisfy.

$$
\begin{aligned}
L_{11} & =\Psi_{1} \bar{A}-N \bar{C}, L_{12}=N+L_{11} \Psi_{2}, \\
L_{13} & =\Psi_{1} \bar{B}, L_{14}=\Psi_{2}
\end{aligned}
$$

Proof: Taking into account the first relation (7) and multiplying it by $\Psi_{1}$, one yields

$$
\Psi_{1} \bar{E} \bar{x}_{k+1}=\Psi_{1} \bar{A} \bar{x}_{k}+\Psi_{1} \bar{B} u_{k}+\Psi_{1} \bar{P} \mu_{k}
$$

Then adding $\Psi_{2} \bar{y}_{k+1}$ to each side of (14) and considering (11), one yields

$\bar{x}_{k+1}=\Psi_{1} \bar{A} \bar{x}_{k}+\Psi_{1} \bar{B} u_{k}+\Psi_{1} \bar{P} \mu_{k}+\Psi_{2} \bar{y}_{k+1}$

It can be seen that system (15) can be modified in the following formula:

$\bar{x}_{k+1}=\Psi_{1} \bar{A} \bar{x}_{k}+\Theta_{k}+\Psi_{1} \bar{P} \mu_{k}$

$\left.\bar{y}_{k}=\bar{C} \bar{x}_{k} 6\right)$

where $\Theta_{k}=\Psi_{1} \bar{B} u_{k}+\Psi_{2} \bar{y}_{k+1}$.

Thus, for system (16), the following observer is designed as:

$$
\begin{aligned}
\hat{\bar{x}}_{k+1} & =\Psi_{1} \bar{A} \hat{\bar{x}}_{k}+\Theta_{k}+N\left(\bar{y}_{k}-\bar{C} \hat{\bar{x}}_{k}\right) \\
& =\Psi_{1} \bar{A} \hat{\bar{x}}_{k}+\Psi_{1} \bar{B} u_{k}+\Psi_{2} \bar{y}_{k+1}+N\left(\bar{y}_{k}-\bar{C} \hat{\bar{x}}_{k}\right)
\end{aligned}
$$

As a result, in equation (17) the term of $\Psi_{2} \bar{y}_{k+1}$ can be omitted by using the auxiliary variable $\xi_{k}=\hat{\bar{x}}_{k}-\Psi_{2} \bar{y}_{k}$. Thus, the observer system in (17) is reconstructed as:

$$
\begin{aligned}
\xi_{k+1} & =\Psi_{1} \bar{A} \hat{\bar{x}}_{k}+\Psi_{1} \bar{B} u_{k}+N\left(\bar{y}_{k}-\bar{C} \hat{\bar{x}}_{k}\right) \\
& =\left(\Psi_{1} \bar{A}-N \bar{C}\right) \xi_{k}+\Psi_{1} \bar{B} u_{k}+\left(N+\left(\Psi_{1} \bar{A}-N \bar{C}\right) \Psi_{2}\right) \bar{y}_{k}
\end{aligned}
$$

The error between the extended state estimate and the real state is expressed as

$\bar{e}_{k+1}=\bar{x}_{k+1}-\hat{\bar{x}}_{k+1}$

Substituting (16) and (17) into (19) the estimation error turns to

$\bar{e}_{k+1}=\left(\Psi_{1} \bar{A}-N \bar{C}\right) \bar{e}_{k}+\Psi_{1} \bar{P} \mu_{k}$

Recalling equations (6) and (12), the term $\Psi_{1} \bar{P}$ is defined as:

$\Psi_{1} \bar{P}=\left[\begin{array}{ll}0_{n \times m} & \varepsilon I_{m}\end{array}\right]^{\mathrm{T}}$

Similar to the manner presented in work [8], the term of $\Psi_{1} \bar{P} \mu_{k}$ in (20) can be neglected if a sufficient small $\varepsilon$ is picked, that means the term $\Psi_{1} \bar{P} \mu_{k}$ is adequately small with respect to the error state vector. Thus, equation (20) can be simplified as

$\bar{e}_{k+1} \cong\left(\Psi_{1} \bar{A}-N \bar{C}\right) \bar{e}_{k}$ 
Finally, considering the auxiliary variable $\hat{\bar{x}}_{k}=\xi_{k}+J \tilde{y}_{k}$, comparing (18) and (8), it can be easily shown that the matrices gains $L_{11}, L_{12}, L_{13}$ and $L_{14}$ are obtained similarly to the equation (13). The proof is ended.

It can be noticed that based on (8) and (11) the extended state estimate $\hat{\bar{x}}_{k}$ is realized. Therefore, the discrete state $x_{k}$ and the disturbance $\mu_{k}$ estimation can be determined as:

$\hat{x}_{k}=\Xi \hat{\bar{x}}_{k}, \quad \hat{\mu}_{k}=\Omega \hat{\bar{x}}_{k}$

where $\Xi=\left[\begin{array}{ll}I_{n} & 0_{n \times m}\end{array}\right]$ and $\Omega=\left[\begin{array}{ll}0_{m \times n} & I_{m}\end{array}\right]$.

The next step is to estimate the non-delayed continuous time state $x(t)$. Thus, the piecewise continuous hybrid system (PCHs) is used. PCHs was first introduced in [14], and then updated in [25]. It consists of two inputs time domain which determine the dynamic of PCHs. The first one refers to the discrete time input and the other to the continuous time input. The discrete time domain $S$ which is called the switching time is indicated by $S=\left\{t_{k}, k=0,1,2, \ldots\right\}$ where $t_{k}$ is the switching instant, and the continuous input is determined by $\Phi_{t}=\{\mathfrak{I}-S\}$, where $\mathfrak{I}=\left\{t \in \mathbb{R}^{+}\right\}$. The PCHs are symbolized as $\sum\left[\left\{k t_{e}\right\}, A_{p}, B_{p}, B_{d}, C_{p}\right]$ with discrete input $v_{d}(t)$ and continuous input $u(t)$ where $A_{p}, B_{p}, B_{d}, C_{p}$ are constant matrices. The switching instants of PCHs are considered sampling instants of the digital camera sensor. Finally, the PCHs as $\sum\left[\{k t e\}, A, B, I_{n}, I_{n}\right]$ with inputs $u_{p}(t)=u(t)$ and $v_{d}(t)=\hat{x}_{k}$ are used. Thus, the continuous time non-delayed state estimate $\hat{x}(t)$ is achieved from the output of the PCHs as:

$$
\hat{x}(t)=e^{A\left(t-t_{k}\right)} x_{k}+\int_{t_{k}}^{t} \exp A(t-\tau) B u(\tau) d \tau
$$

\section{CSO Stability Analysis}

In the observation design, the estimation error is defined as a difference between the real state, and its estimation and it is denoted as $e(t)$. Thus, the $\mathrm{CSO}$ is determined as

$e(t)=x(t)-\hat{x}(t)$

Recalling equation (24), the real-time system state is defined similarly as follows:
$x(t)=e^{A\left(t-t_{k}\right)} x_{k}+\int_{t_{k}}^{t} \exp A(t-\tau) B u(\tau) d \tau$

Substituting (24) and (26) into (25), one yields

$$
e(t)=e^{A\left(t-k t_{e}\right)} e_{k}
$$

where $e_{k}=x_{k}-\hat{x}_{k}$ is the error of the discrete system state estimate. Taking into account the estimation errors in (19) the estimation error $\bar{e}_{k}$ can be written as follows.

$\bar{e}_{k}=\bar{x}_{k}-\hat{\bar{x}}_{k}$

$$
=\left[\begin{array}{c}
e_{k} \\
e_{\hat{\mu}_{k}}
\end{array}\right]
$$

where $e_{\hat{\mu}_{k}}$ is the estimation error of the disturbance.

Before designing the observer gain $N$ that guarantees that $\Psi_{1} \bar{A}-N \bar{C}$ is Schur and the pair $\left(\Psi_{1} \bar{A}, \bar{C}\right)$ needs to be detectable. Thus, to verify that, the Lemma 1 is considered, the pair $\left(\Psi_{1} \bar{A}, \bar{C}\right)$ is detectable if the following rank condition is satisfied

$\operatorname{rank}\left[\begin{array}{c}\bar{C} \\ \lambda I_{n+m}-\Psi_{1} \bar{A}\end{array}\right]=n+m \quad$ for all $\lambda \in \mathbb{C}$

Remind that

$$
\begin{aligned}
& \operatorname{rank}\left[\begin{array}{c}
\bar{C} \\
\lambda I_{n+m}-\Psi_{1} \bar{A}
\end{array}\right]=\operatorname{rank}\left[\begin{array}{cc}
C_{d} & I_{m} \\
-C_{d} \Phi & (\lambda+\varepsilon) I_{m} \\
\lambda I_{n}-\Phi & 0_{n \times m}
\end{array}\right] \\
& =\operatorname{rank}\left[\begin{array}{ccc}
0 & I_{m} & I_{m} \\
C_{d} & -\lambda I_{m} & (\lambda+\varepsilon) I_{m} \\
I_{n} & 0_{n \times m} & 0_{n \times m}
\end{array}\right]\left[\begin{array}{cc}
0_{m} & I_{m} \\
C_{d} & 0_{m} \\
\lambda I_{n}-\Phi & 0_{n \times m}
\end{array}\right]
\end{aligned}
$$

Notice that for $\forall|\lambda|=\varepsilon$ one gets

$$
\operatorname{rank}\left[\begin{array}{c}
\bar{C} \\
\lambda I_{n+m}-\Psi_{1} \bar{A}
\end{array}\right]=\operatorname{rank}\left[\begin{array}{c}
C_{d} \\
\lambda I_{n}-\Phi
\end{array}\right]+m
$$

Considering equations (30) and (31), then (29) is the same conditions of equation (3).

Thus, the pair $\left(\Psi_{1} \bar{A}, \bar{C}\right)$ is detectable, and the observer gain is designed based on LMI which requires the following Lemma. 
Lemma 2 [3]: Schur complement is described as follows:

$$
\left[\begin{array}{ll}
Q_{0} & S_{0} \\
S_{0}^{T} & R_{0}
\end{array}\right]<0
$$

and is equivalent to $R_{0}<0, Q_{0}-S_{0} R_{0}^{-1} S_{0}^{T}<0$, where $R_{0}$ and $Q_{0}$ are symmetric.

Theorem 2: The system of dynamic errors provided in (22) are stable if there exists a symmetric positive definite matrix $P \in \mathbb{R}^{(n+m) \times(n+m)}$ which satisfies algebraic Lyapunov inequality

$$
\left[\begin{array}{cc}
-P & \left(P \Psi_{1} \bar{A}-Y \bar{C}\right)^{T} \\
(*) & -P
\end{array}\right]<0
$$

where $(*)$ in a matrix denotes the asymmetrical entry, and the proposed observer gain is designed as $N=P^{-1} Y$.

Proof: Let the Lyapunov function be defined as follows:

$$
V_{o}\left(\bar{e}_{k}\right)=\left(\bar{e}_{k}\right)^{T} P \bar{e}_{k}
$$

Recalling the estimation error in systems (22) and (34), one gets:

$$
\Delta V_{o}\left(\bar{e}_{k}\right)=V_{o}\left(\bar{e}_{k+1}\right)-V_{o}\left(\bar{e}_{k}\right)<0, \quad \forall \bar{e}_{k} \in \mathbb{R}^{n+m}
$$

Therefore

$$
\begin{aligned}
& \Delta V_{o}\left(\bar{e}_{k}\right)=\left(\bar{e}_{k+1}\right)^{T} P \bar{e}_{k+1}-\left(\bar{e}_{k}\right)^{T} P \bar{e}_{k} \\
& \Delta V_{o}\left(\bar{e}_{k}\right)=\left(\left[\Psi_{1} \bar{A}-N \bar{C}\right] \bar{e}_{k}\right)^{T} P\left(\left[\Psi_{1} \bar{A}-N \bar{C}\right] \bar{e}_{k}\right) \\
& -\left(\bar{e}_{k}\right)^{T} P \bar{e}_{k} \\
& =\left(\bar{e}_{k}\right)^{T}\left(\left(\Psi_{1} \bar{A}-N \bar{C}\right)^{T} P\left(\left(\Psi_{1} \bar{A}-N \bar{C}\right) \bar{e}_{k}\right)-P \bar{e}_{k}\right) \\
& \Delta V\left(\bar{e}_{k}\right)<0 \Leftrightarrow\left(\Psi_{1} \bar{A}-N \bar{C}\right)^{T} P\left(\Psi_{1} \bar{A}-N \bar{C}\right)-P<0 \\
& =\left(\bar{e}_{k}\right)^{T}\left(\left(\Psi_{1} \bar{A}-N \bar{C}\right)^{T} P\left(\Psi_{1} \bar{A}-N \bar{C}\right)-P\right) \bar{e}_{k}<0 \\
& \Leftrightarrow\left(\Psi_{1} \bar{A}-N \bar{C}\right)^{T} P\left(\Psi_{1} \bar{A}-N \bar{C}\right)-P
\end{aligned}
$$

Remark 1: The observer gain $N$ guarantees that the matrix $\Psi_{1} \bar{A}-N \bar{C}$ is Schur. It can be noticed that the estimation error $\bar{e}_{k}$ in equation (28) is composed of two vectors errors $e_{k}$ and $e_{\mu_{k}}$. As a result, the error in equation (28) is stable; this means that the error $e(t)$ which is well-defined in (27) settles down to zero asymptotically.
In order to simplify the proposed observer design method, a designing procedure is concisely summarized as follows

- Set the continuous system of equation (1) into the form of an augmented descriptor system as equation (7) with the parameters defined in equation (6).

- Set the matrices $\Psi_{1}$ and $\Psi_{2}$ from equation (12) to satisfy the necessary condition of equation (11).

- Solve the equation (33) by LMI to obtain the gain $N=P^{-1} Y$, and then use $N$ to compute the other observer parameters as provided in equation (13).

- Obtain the state estimation $\hat{x}_{k}$ and $\hat{\mu}_{k}$ from the equation (23).

- Finally, set the PCHs as $\sum\left[\{k t e\}, A, B, I_{n}, I_{n}\right]$ with inputs $v_{d}(t)=\hat{x}_{k}$ and $u_{p}(t)=u(t)$ then obtain the continuous system state without delay from equation (24).

\section{Numerical Simulation Results}

To investigate the CSO performance, the CSO is compared with Chain Observer ( $\mathrm{CO}$ ) which is introduced in [20]. The comparison has been performed in MATLAB Simulink for the similar system which was addressed in [20] without measurement noise. This comparison considers the same system with measurement noise which is defined as follows:

$\left\{\begin{array}{l}\dot{x}(t)=\left[\begin{array}{ll}0 & 1 \\ a & b\end{array}\right] x(t)+\left[\begin{array}{l}0 \\ c\end{array}\right] u(t) \\ y=\left[\begin{array}{ll}1 & 0\end{array}\right] x(t) \\ \bar{y}(t)=y^{*}(t-d)+v_{k}\end{array}\right.$

where $a, b$ and $c$ equals $-3,-1$ and 1 , respectively. The initial conditions for the system (36): Chain observer and the proposed observer are $x(0)=\left[\begin{array}{ll}2 & -4\end{array}\right]^{T}, \hat{x}(0)=\left[\begin{array}{ll}0 & 0\end{array}\right]^{T}$, and $\hat{\bar{x}}(0)=\left[\begin{array}{lll}0 & 0 & 0\end{array}\right]^{T}$ , respectively. The considered control signal is $6 \sin t+6 \sin 3 t$ as it is shown in Figure 2. The value of the sampling period is $t_{e}=20 \mathrm{~ms}$. The Chain observer gain $K$ is selected such that the eigenvalues of the matrix $A_{m}$ are at -10 and-15, respectively. where $A_{m}=A-K C$. To compute the continuous state observer gain $N$, the constant $\varepsilon$ is chosen as $1 \times 10^{-5}$ and the matrices $\Psi_{1}$ and $\Psi_{2}$ are achieved from (12) as: 


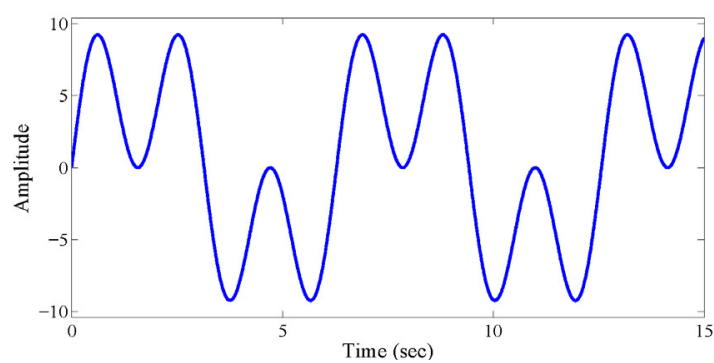

Figure 2. The control signal

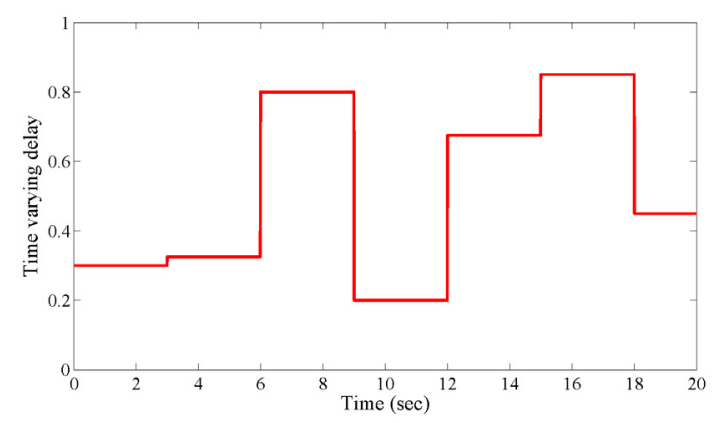

Figure 3. The piecewise constant delays

$$
\Psi_{1}=\left[\begin{array}{ccc}
1 & 0 & 1 \\
0 & 1 & 0 \\
-1 & 0 & 1
\end{array}\right], \quad \Psi_{2}=\left[\begin{array}{l}
0 \\
0 \\
1
\end{array}\right]
$$

The CSO gain $N$ is obtained by solving the LMI of equation (33) in MATLAB which is $N=\left[\begin{array}{lll}0.4997 & -0.0297 & -0.4997\end{array}\right]^{T}$, and then the other observer gains are obtained from (13) as follows:

$$
\begin{aligned}
& L_{11}=\left[\begin{array}{ccc}
0.4997 & 0.0198 & -0.4997 \\
-0.0297 & 0.9414 & 0.0297 \\
-0.4997 & -0.0198 & 0.4997
\end{array}\right], L_{12}=10^{-5} \times\left[\begin{array}{c}
0 \\
0 \\
-1
\end{array}\right] \\
& L_{13}=\left[\begin{array}{c}
0.0002 \\
0.0198 \\
-0.0002
\end{array}\right], L_{14}=\Psi_{2}
\end{aligned}
$$

It is worth mentioning that the comparison between CSO and CO has been derived in the case of the piecewise constant delay which was addressed in [20] as well as the varying time delay without and with measurement noise as presented in the following subsections.

\subsection{The Piecewise Constant Delays}

In this section, the CSO is verified to the piecewise constant delays which were illustrated in an ideal condition by Chain observer in [20]. The considered piecewise constant delay is in the range of $d \in(0.1,1) s$ as shown in Figure 3.

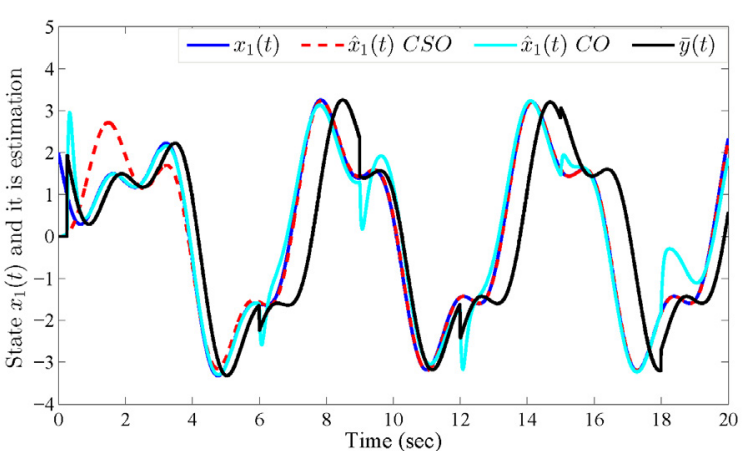

(a)

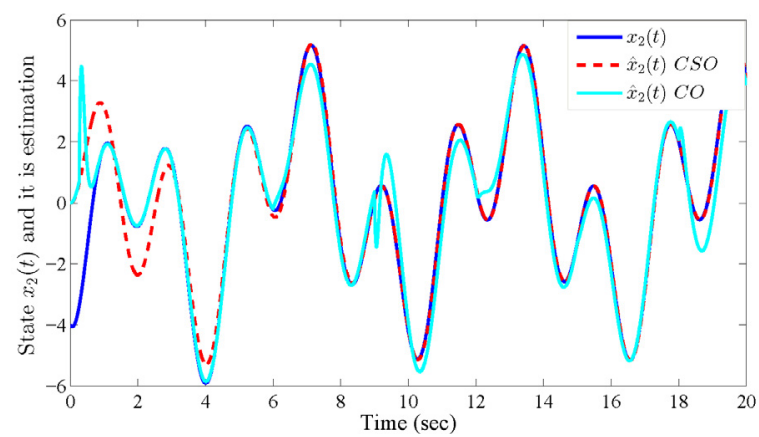

(b)

Figure 4. Observer's performance under piecewise constant delays. (a) $x_{1}(t)$ and its estimation, (b) $x_{2}(t)$ and its estimation

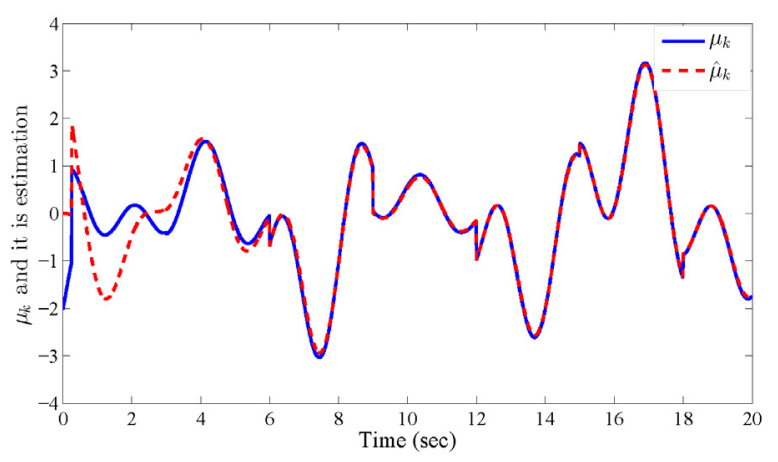

Figure 5. The disturbance $\mu_{k}$ and its estimation

Remark 2: It can be noticed that the piecewise constant delays in Figure 3 are completely known for the Chain observer, while the time delay for the proposed observer is unknown. This means that the proposed observer considers the piecewise constant delays as the particular case of unknown delayed measurements.

Figure 4 illustrates the equivalent results and the results demonstrate that the performance of the $\mathrm{CO}$ is smooth from $t=0$ to $t=6 \mathrm{~s}$. This occurs because the variation in the time delay is small, after $t=6 \mathrm{~s}$ the change in the magnitude of the time delay is larger. 
In the transient response of $\mathrm{CO}$, the performance shows significant overshoots, which accommodate the system state changes due to the timevarying delays, while the CSO estimates well the continuous system state and the observer gain $N$ in (33) guarantees fast convergence response without overshoots. Furthermore, the CSO estimates the disturbance $\mu_{k}$ as provided in equation (23). As expected result, an accurate performance is obtained as shown in Figure 5.

\subsection{The Time-Varying Delay with Measurement Noise}

In this section, the simulation has been investigated in the case of the unknown time-varying delay with measurement noise which makes it possible to validate the simulation in real environmental conditions. Therefore, it is assumed that the delay is an unknown time-varying as presented in Figure 6. In order to show the proposed observer performance under the measurement noise, the Gaussian noise signal with zero mean value and variance 7 is applied to the vision sensor as introduced in equation (1c), and the Gaussian noise is demonstrated in Figure 7. The obtained results are shown in Figure 8. It can be easily noticed that the proposed observer estimated the non-delayed continuous system state as accurately as expected, while the Chain observer is strongly affected by the fasting variation of the time delays values which were defined as observation accumulation errors in [20]. The results are that the Gaussian measurement noise is added to the disturbance $\mu_{k}$ with time-varying delay, and the effect of $\mu_{k}$ is attenuated by the chosen small value of $\varepsilon$ as stated in equation (21). As expected, the results in Figure 8 show that the proposed observer under the effect of the Gaussian measurement noise estimated the non-delayed continuous state to the acceptable accuracy level and provided better performance than the Chain observer. As presented in Figure 9, one can achieve: the continuous state observer is robust to measurement noise and the proposed observer estimates well the disturbance in the existence of measurement noise.

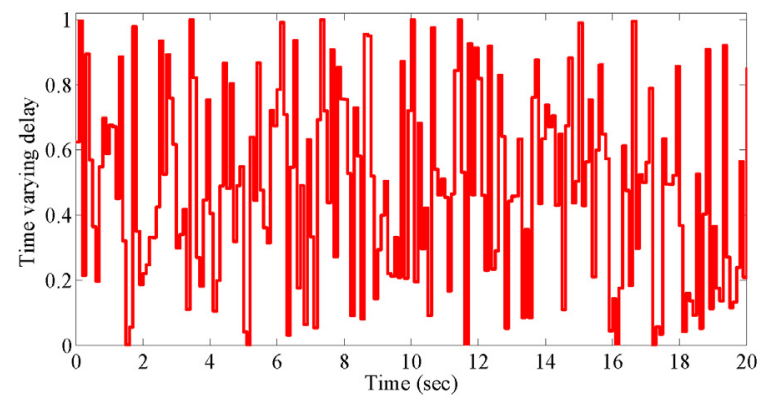

Figure 6. The time-varying delay

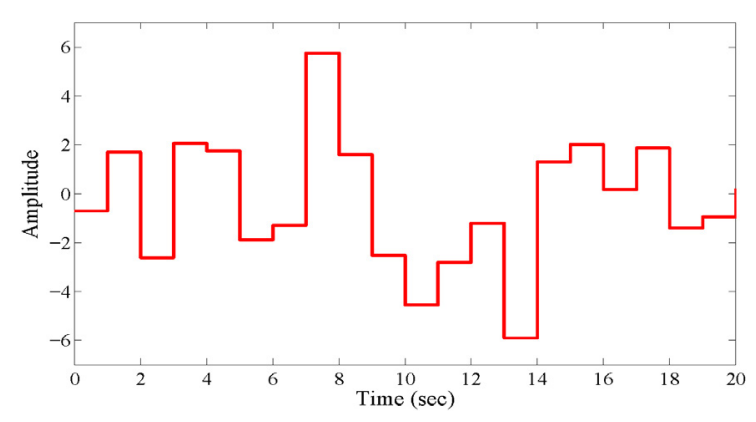

Figure 7. The Gaussian measurement noise



(a)

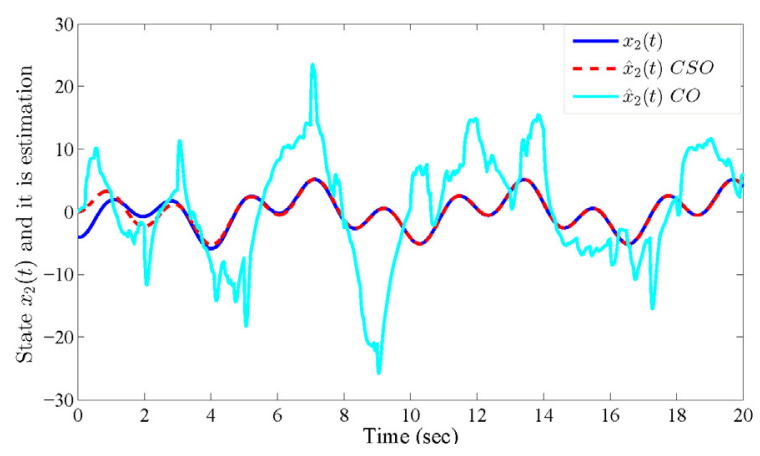

(b)

Figure 8. Observer's performance under timevarying delays subject to measurement noise. (a) $x_{1}(t)$ and its estimation, (b) $x_{2}(t)$ and its estimation

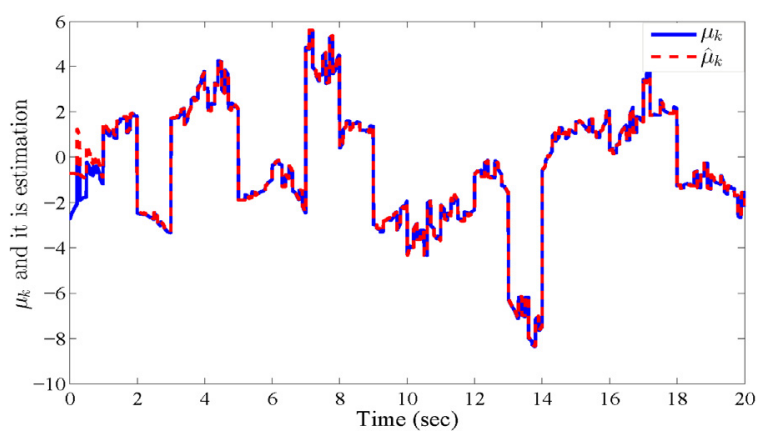

Figure 9. The disturbance $\mu_{k}$ and its estimation 


\section{Conclusion}

This paper studies the NVSCS with sampled and unknown time-varying delayed measurement problem subject to measurement noise. The continuous state observer is proposed to estimate the non-delayed continuous system state from the output measurement which is available in sampled and unknown time-varying delayed measurements subject to measurement noise. The gain that guarantees the estimation errors which asymptotically stable are designed by a linear matrix inequality. The CSO technique is assessed via a comparison with the Chain observer. As the obtained simulation results demonstrate the continuous state observer estimated the continuous system state without delay and the disturbance accurately from the available measurement in the form of sampled and unknown time-varying delayed

\section{REFERENCES}

1. Ahmed-Ali, T., Cherrier, E. \& Lamnabhi Lagarrigue, F. (2012). Cascade High Gain Predictors for A class of Nonlinear Systems, IEEE Transactions on Automatic Control, 57(1), 221-226.

2. Ahrens, J. H., Tan, X. \& Khalil, H. K. (2009). Multirate Sampled-Data Output Feedback Control with Application to Smart Material Actuated Systems, IEEE Transactions on Automatic Control, 54(11), 2518.

3. Boyd, S., El Ghaoui, L., Feron, E. \& Balakrishnan, V. (1994). Linear Matrix Inequalities in System and Control Theory. SIAM.

4. Chen, G. P., Li, J. M. \& Zhang, L. G. (2005). Design of Observers for A class of Hybrid Systems with Time Delay, Computer Integrated Manufacturing Systems, 11(6), 891.

5. Duviella, E., Chiron, P. \& Charbonnaud, P. (2008). Networked Hydrographical Systems: A Reactive Control Strategy Integrating Time Transfer Delays, Studies in Informatics and Control, 17(2), 153.

6. François, C. \& Seth, H. (2006). Visual Servo Control. I. Basic Approaches, IEEE Robotics \& Automation Magazine, 13(4), 82-90.

7. Fridman, E., Seuret, A. \& Richard, J. P. (2004). Robust Sampled-Data Stabilization of Linear Systems: An Input Delay Approach, Automatica, 40(8), 1441-1446. signal under the condition of measurement noise. In addition, the continuous state observer illustrates a better performance than the Chain observer. Moreover, the continuous state observer provided robust performance to the measurement noise.

\section{Acknowledgments}

This work is partially supported by the National Natural Science Foundation of China under grant 61773212, 61304077; the International Science \& Technology Cooperation Program of China under grant 2015DFA01710; the Natural Science Foundation of Jiangsu Province under grant BK20170094; the Chinese Ministry of Education Project of Humanities and Social Sciences under grant 13YJCZH171; the 11th Jiangsu Province Six talent peaks of high-level talents project under grant 2014ZBZZ005.

8. Gao, Z. (2015). Estimation and Compensation for Lipschitz Nonlinear Discrete-Time Systems Subjected to Unknown Measurement Delays, IEEE Transactions on Industrial Electronics, 62(9), 5950-5961.

9. Gortcheva, E., Garrido R., Gonzalez, E. \& Carvallo, A. (2001). Predicting A moving Object Position for Visual Servoing: Theory and Experiments, International Journal of Adaptive Control and Signal Processing, 15(4), 377-392.

10. Guerra, R. V., Rubio, J. M., Cuéllar, B. M. \& Sánchez, G. I. D. (2016). Dynamic Delayed Controllers for Unstable Recycling Systems with Time Delays, Studies in Informatics and Control, 25(2), 195-206.

11. Hussein, T. \& Söffker, D. (2012). State Variables Estimation of Flexible Link Robot using Vision Sensor Data. In International Conference on Mathematical Modelling (pp. 193-198).

12. Idaku, I., Yoshihiro, N. \& Masatoshi, I. (1996). Target Tracking Algorithm for $1 \mathrm{~ms}$ Visual Feedback System using Massively Parallel Processing. In IEEE International Conference on Robotics and Automation (pp. 2309-2314).

13. Jean, J.-H. \& Lian, F.-L. (2012). Robust Visual Servo Control of A mobile Robot for Object Tracking using Shape Parameters, IEEE Transactions on Control Systems Technology, 20(6), 1461-1472. 
14. Koncar, V. \& Vasseur, C. (2003). Control of Linear Systems using Piecewise Continuous Systems, IET Control Theory \& Applications, $150(6), 565-576$.

15. Mohammed, A., Wang, H. \& Tian, Y. (2017). Feedback Delay Compensation of A visual Servoing System using A piecewise Continuous and Current Estimator-Based Observer, Turkish Journal of Electrical Engineering \& Computer Sciences, 25(5), 3738-3751.

16. Peter, I. C. \& Malcolm, C. G. (1996). Dynamic Effects in Visual Closed-Loop Systems, IEEE Transactions on Robotics and Automation, 12(5), 671-683.

17. Seth, H., Gregory, D. H. \& Peter, I. C. (1996). A tutorial on Visual Servo Control, IEEE Transactions on Robotics and Automation, 12(5), 651-670.

18. Seuret, A., Michaut, F., Richard, J. P. \& Divoux, T. (2006). Networked Control using GPS Synchronization. In American Control Conference (pp. 6-pp).

19. Shaked, U. \& Fridman, E. (2002). A descriptor System Approach to $H_{\infty}$ Control of Linear TimeDelay Systems, IEEE Transactions on Automatic Control, 47(2), 253-270.

20. Subbarao, K. \& Muralidhar, P. C. (2009). State Observer for Linear Systems with Piece-wise Constant Output Delays, IET Control Theory \& Applications, 3(8), 1017-1022.
21. Wang, H. P., Vasseur, C., Christov, N. \& Koncar, V. (2012). Vision Servoing of Robot Systems using Piecewise Continuous Controllers and Observers, Mechanical Systems and Signal Processing, 33, 132-141.

22. Wang, H. P., Tian, Y. \& Christov, N. (2016). Piecewise-Continuous Observers for Linear Systems with Sampled and Delayed Output, International Journal of Systems Science, 47(8), 1804-1815

23. Wang, H. P., Vasseur, C., Koncar, V., Chamroo, A. \& Christov, N. (2010). Sampled Tracking for Delayed Systems using Two Time-Scales Sampled-Data Controllers, Studies in Informatics and Control, 19(4), 339-346.

24. Wang, H. P., Tian, Y. \& Vasseur, C. (2015). Piecewise Continuous Hybrid Systems Based Observer Design for Linear Systems with Variable Sampling Periods and Delay Output, Signal Processing, 114, 75-84.

25. Wang, H., Vasseur, C., Tian, Y., Koncar, V. \& Christov, N. (2011). Recursive Model Free Controller: Application to Friction Compens ation and Trajectory Tracking, International Journal of Control, Automation and Systems, 9(6), 1146-1153.

26. $\mathrm{Wu}$, H., Lou, L., Chen, C. C., Hirche, S. \& Kuhnlenz, K. (2013). Cloud-Based Networked Visual Servo Control, IEEE Transactions on Industrial Electronics, 60(2), 554-566.

27. Zhang, J., Lin, Y. \& Shi, P. (2015). Output Tracking Control of Networked Control Systems Via Delay Compensation Controllers, Automatica, 57, 85-92. 